\title{
Variational principle of stationary action for fractional nonlocal media and fields
}

\author{
Vasily E. Tarasov
}

\begin{abstract}
Derivatives and integrals of non-integer orders have a wide application to describe complex properties of physical systems and media including nonlocality of power-law type and long-term memory. We suggest an extension of the standard variational principle for fractional nonlocal media with power-law type nonlocality that is described by the Riesz-type derivatives of non-integer orders. As examples of application of the suggested variational principle, we consider an $\mathrm{N}$-dimensional model of waves in anisotropic fractional nonlocal media, and a one-dimensional continuum (string) with power-law spatial dispersion. The main advantage of the suggested fractional variational principle is that it is connected with microstructural lattice approach and the lattice fractional calculus, which is recently proposed.
\end{abstract}

PACS: $45.10 . \mathrm{Hj} ; 04.20 . \mathrm{Fy}$

MSC: 26A33; 34A08; 49S05

\section{Introduction}

Derivatives and integrals of non-integer orders [11, 13, 23, $42,43]$ have wide application in physics and mechanics $[6,14,22,29,30]$. The tools of fractional derivatives and integrals allow us to investigate the behavior of materials and systems that are characterized by power-law nonlocality, power-law long-term memory and fractal properties. There are different types of fractional derivatives such as Riemann-Liouville, Riesz, Grünwald-Letnikov, Caputo, Marchaud, Hadamard, Weyl and others [11, 23]. Moreover, all these different fractional derivatives are related to each other. For example, the Grünwald-Letnikov derivatives coizncide with Marchaud derivatives for wide class of functions (see Sections 20.2 and 20.3 in [23]), and the Marchaud derivatives are connected with the Liouville derivatives (see Section 5.4 in [23]). All fractional derivatives have a lot of unusual properties that lead to great difficulties in application of fractional calculus. Therefore question about what type of fractional derivatives should be used in applications is not a simple question. Selection of the type of fractional derivative is largely dictated by the properties of objects and materials under consideration.

Correspondence: tarasov@theory.sinp.msu.ru

Skobeltsyn Institute of Nuclear Physics, Lomonosov Moscow State University, Moscow 119991, Russia
Nonlocal continuum theory $[8,21]$ is based on the assumption that the forces between particles of continuum have long-range type thus reflecting the long-range character of inter-atomic forces. If the nonlocality has a power-law type then we can use the fractional calculus for nonlocal continuum mechanics. It is important to apply such types of fractional derivatives that allow us to take into account the long-range particle interactions in microstructural models. Therefore in fractional nonlocal theory, we should use the fractional-order derivatives that are directly connected with models of lattices with longrange interactions. The microstructural lattice approach, which includes consideration of a continuum limit, allows us to select a type of fractional derivatives that will adequately and correctly describe fractional nonlocal continua.

Fractional nonlocal continuum mechanics is an area of continuum mechanics concerned with the behavior of continua with nonlocalities of power-law types that are described by using the theory of derivatives and integrals of non-integer orders. In phenomenological approach, one of the methods to describe the fractional nonlocal continua is based on the variational principle with Lagrangian density that contains fractional derivatives with respect to coordinates. A generalization of traditional

\section{Springer}

(c) 2015 Tarasov Open Access This article is distributed under the terms of the Creative Commons Attribution 4.0 International License (http://creativecommons.org/licenses/by/4.0/), which permits unrestricted use, distribution, and reproduction in any medium, provided you give appropriate credit to the original author(s) and the source, provide a link to the Creative Commons license, and indicate if changes were made. 
calculus of variations for systems that are described by the Riemann-Liouville fractional derivatives has been suggested by Agrawal in [1]. Subsequent by extensions of variational calculus for the Riemann-Liouville derivatives [5] and other type of fractional derivatives such as the Caputo derivative $[15,18,19]$, the Hadamard derivative [3], the Riesz derivatives [2], and fractional integrals [4] have been derived. It should be noted that the extension of variational calculus for the Riesz derivatives, suggested in [2], is really derived for so-called the Riesz-RiemannLiouville and the Riesz-Caputo derivatives rather than the usual Riesz fractional derivatives [23]. Unfortunately all suggested extensions of variational calculus are not connected with the microstructural lattice approach. Therefore, an important problem is to formulate a fractional variational principle compatible with the lattice approach.

Let us explain our main motivation to suggest new variational principle with fractional-order derivatives. Recently, the lattice fractional calculus and the lattice fractional derivatives have been suggested in [37, 39]. In the continuum limit the suggested lattice derivatives are transformed into continuum fractional derivatives of the Riesz type [39]. The Riesz-type fractional derivative $R T \mathbb{D}_{j}^{\alpha}$ is a derivative with respect to one coordinate $x_{j} \in \mathbb{R}^{1}$ instead of the usual Riesz derivative that is a fractional generalization of $N$-dimensional Laplacian [11, 20, 23]. The Riesz-type operator ${ }^{R T} \mathbb{D}_{j}^{\alpha}$ can be considered as a partial derivative of non-integer order. Taking into account the direct connection of the Riesz-type derivatives with microstructural lattice approach, we suggest to use these fractional derivatives to formulate new fractional variational principle that is compatible with the microstructural lattice approach.

In addition to "lattice" motivation, it is useful to have a variational principle that allows us to derive fractional differential equations of motion that can be solved for a wide class of Lagrangian densities. It is known that the Riemann-Liouville, Caputo, Liouville, Marchaud derivatives are defined in the left-sided and the right-sided forms. In general, we should take into account these two forms in the Lagrangian density if we use these derivatives. The corresponding fractional Euler-Lagrange equations contain the left-sided and the right-sided fractional derivatives as well. In addition, the integration by parts, which is used in derivation of the Euler-Lagrange equations from the variational principle, transforms the left-sided derivatives into the right-sided and vice versa [23]. As a result, we get a mixture of the left- and rightsided derivatives in equations of motion. Unfortunately, these equations can be solved only for a narrow class of examples. In this paper, we suggest a fractional variational principle based on the Riesz-type fractional derivatives $[37,39]$ that are modified Riesz fractional derivatives
[11, 20, 23], and have no left- and the right-sided forms. In addition, the integration by parts transforms the Riesz fractional derivatives into itself. The corresponding fractional Euler-Lagrange equations can be solved for a wide class of Lagrangian densities by methods described in [11].

It should be emphasized that the main advantage of the suggested variational approach is that the proposed Riesz-type fractional derivatives naturally arise in the fractional continuum mechanics based on the lattice models [31-34, 40], and are directly connected with lattice fractional derivatives suggested in $[37,39]$.

As examples, we consider $N$-dimensional model of waves in anisotropic fractional nonlocal media and then we demonstrate that this model is connected with microstructural lattice model. We also consider an elastic string, which is made of a material with a spatial dispersion of power-law type, i.e., string with fractional nonlocal material. Using the suggested variational principle, we derive the fractional differential equations and then some solutions of these equations are obtained.

\section{Fractional derivatives and integration by parts}

\subsection{Marchaud fractional derivatives}

In order to derive equations of motion from a fractional variational principle, we should use the integration by parts. Unfortunately the integration by parts for most of fractional derivatives transforms the left-sided derivatives into the right-sided and vice versa. Let us give an example.

Definition 1. The left-sided (+) and right-sided (-) Marchaud fractional derivatives of order $\alpha \in(0 ; 1)$ (see Section 5.4 of [23]) are defined by the equation

$$
\begin{aligned}
\left({ }^{M} D_{x, \pm}^{\alpha} f\right)(x):= & \frac{\alpha}{\Gamma(1-\alpha)} \int_{0}^{\infty} \frac{f(x)-f(x \mp z)}{z^{\alpha+1}} d z, \\
& (0<\alpha<1, \quad x \in \mathbb{R}) .
\end{aligned}
$$

The Marchaud fractional derivatives of orders $\alpha>1$ (see Section 5.6 of [23]) are defined as

$$
\left({ }^{M} D_{x, \pm}^{\alpha} f\right)(x):=\frac{\alpha}{\Gamma(1-\alpha)} \frac{1}{A_{m}(\alpha)} \int_{0}^{\infty} \frac{\left(\Delta_{ \pm z}^{m} f\right)(x)}{z^{1+\alpha}} d z
$$

where $m$ is an integer greater than $\alpha$, and

$$
\begin{aligned}
\left(\Delta_{z}^{m} f\right)(x) & :=\sum_{n=0}^{m} \frac{(-1)^{n} m !}{n !(m-n) !} f(x-n z), \\
A_{m}(\alpha) & :=\sum_{n=0}^{m} \frac{(-1)^{n} m !}{n !(m-n) !} n^{\alpha} .
\end{aligned}
$$


Remark 1. Note that the right hand side of (2) does not depend on $m$ if $m$ is an even integer number greater than $\alpha$ (for example, $m=2[\alpha / 2]+2$ ).

Remark 2. For $\alpha=1,2, \ldots m-1$, the expression $\Gamma(1-$ $\alpha) A_{m}(\alpha)$ is given (see Eq. 5.81 of [23]) by

$$
\frac{\Gamma(1-\alpha) A_{m}(\alpha)}{\alpha}=\int_{0}^{\infty} \frac{1-e^{-z}}{z^{1+\alpha}} d z=\frac{m}{\alpha} \int_{0}^{1} \frac{(1-s)^{m-1}}{(\ln (1 / s))^{\alpha}} .
$$

Remark 3. It is important to note that the GrünwaldLetnikov fractional derivatives coincide with the Marchaud fractional derivatives (see Section 20.3 in [23]) for functions from the space $L_{r}(\mathbb{R})$, where $1 \leqslant r<\infty$ (see Theorem 20.4 in [23]). Moreover both the GrünwaldLetnikov and Marchaud derivatives have the same domain of definition.

Proposition 1. For the Marchaud fractional derivatives, the integration by parts has the form

$$
\int_{-\infty}^{+\infty} f(x)\left({ }^{M} D_{x,+}^{\alpha} g\right)(x) d x=\int_{-\infty}^{+\infty}\left({ }^{M} D_{x,-}^{\alpha} f\right)(x) g(x) d x .
$$

Equation (6) holds for functions $f(x) \in L_{s}(\mathbb{R})$, $g(x) \in L_{t}(\mathbb{R})$, such that $\left({ }^{M} D_{x,-}^{\alpha} f\right)(x) \in L_{p}(\mathbb{R})$ and $\left({ }^{M} D_{x,+}^{\alpha} g\right)(x) \in L_{r}(\mathbb{R})$, where $p>1, r>1, p^{-1}+r^{-1}=$ $1+\alpha, s^{-1}=p^{-1}-\alpha$, and $t^{-1}=r^{-1}-\alpha$.

Proof. This proposition is proved in Section 6.3 of [23] (see Equation 6.27 of [23]).

\subsection{Riesz-type fractional derivatives}

Let us now define the fractional derivatives of the Riesz types for $\mathbb{R}^{N}$. We will use the Cartesian coordinate system with the basis vectors $\mathbf{e}_{j}(j=1,2, \ldots, N)$, and the radius vector $\mathbf{r}=\sum_{j=1}^{N} x_{j} \mathbf{e}_{j}$. The fractional derivatives of the Riesz types have been introduced in [37,39].

Definition 2. The Riesz-type fractional derivative of order $\alpha$ is defined by the equation

$$
\begin{aligned}
R T \mathbb{D}_{j}^{\alpha} f(\boldsymbol{r}):= & \frac{1}{d_{1}(m, \alpha)} \int_{-\infty}^{+\infty} \frac{1}{\left|z_{j}\right|^{\alpha+1}}\left(\Delta_{z_{j}}^{m} f\right)(\boldsymbol{r}) d z_{j}, \\
& \times(0<\alpha<m),
\end{aligned}
$$

where $\left(\Delta_{z_{j}}^{m} u\right)(r)$ is a finite difference of order $m$ of a function $f(\boldsymbol{r})$ with the vector step $\boldsymbol{z}_{j}=z_{j} \boldsymbol{e}_{j} \in \mathbb{R}^{N}$ for the point $r \in \mathbb{R}^{N}$. The centered difference

$$
\left(\Delta_{z j}^{m} f\right)(\boldsymbol{r})=\sum_{n=0}^{m}(-1)^{n} \frac{m !}{n !(m-n) !} f\left(\boldsymbol{r}+(m / 2-n) z_{j} \boldsymbol{e}_{j}\right) .
$$

The constant $d_{1}(m, \alpha)$ is defined as

$$
d_{1}(m, \alpha)=\frac{\pi^{3 / 2} A_{m}^{c}(\alpha)}{2^{\alpha} \Gamma(1+\alpha / 2) \Gamma((1+\alpha) / 2) \sin (\pi \alpha / 2)},
$$

where

$$
A_{m}^{c}(\alpha)=2 \sum_{s=0}^{[m / 2]}(-1)^{s-1} \frac{m !}{s !(m-s) !}(m / 2-s)^{\alpha}
$$

for the centered difference (8).

Remark 4. The constant $d_{1}(m, \alpha)$ depends only on $m$ and $\alpha$. It is different from zero for all $\alpha>0$ in the case of an even $m$ (see Theorem 26.1 in [23]). Note that the integral (7) does not depend on the choice of $m>\alpha$. Therefore, we can always choose an even integer $m$, so that it is greater than parameter $\alpha$, and we can use the centered difference (8) for all positive real values of $\alpha$.

Remark 5. It should be noted that we can use the noncentered difference instead of the centered difference (8). The non-centered difference is defined by the equation

$$
\left(\Delta_{z j}^{m} f\right)(\mathbf{r})=\sum_{n=0}^{m}(-1)^{n} \frac{m !}{n !(m-n) !} f\left(\mathbf{r}-n z_{j} \mathbf{e}_{j}\right),
$$

and the corresponding coefficient $A_{m}^{n c}(\alpha)$ instead of $A_{m}^{c}(\alpha)$, where

$$
A_{m}^{n c}(\alpha)=\sum_{s=0}^{m}(-1)^{s-1} \frac{m !}{s !(m-s) !} s^{\alpha} .
$$

In the case of non-centered differences the constant $d_{1}(m, \alpha)$ vanishes if and only if $\alpha=1,3,5, \ldots, 2[m / 2]-1$. Therefore the non-centered differences (9) can be used only for non-integer positive orders $\alpha$ and for odd integer values of $\alpha$.

Remark 6. Using (7), we can see that the Riesz-type fractional derivative ${ }^{R T} \mathbb{D}_{j}^{\alpha_{j}} f(\mathbf{r})$ is the Riesz derivative [23] of the function $f(\mathbf{r})$ with respect to one coordinate $x_{j} \in \mathbb{R}^{1}$ instead of the usual Riesz operator defined for the vector $\mathbf{r} \in \mathbb{R}^{N}$ as a fractional generalization of $N$-dimensional Laplacian. The Riesz-type fractional derivative $R T \mathbb{D}_{j}^{\alpha_{j}}$ is the Riesz fractional derivative with respect to $x_{j}$ for $\mathbb{R}^{1}$. The Riesz-type operator $R T \mathbb{D}_{j}^{\alpha}$ can be considered as a partial derivative of non-integer order.

Remark 7. In $[37,39]$ the Riesz-type fractional derivative ${ }^{R T} \mathbb{D}_{j}^{\alpha}$ is denoted as $\mathbb{D}_{C}^{+}\left[\begin{array}{l}\alpha \\ j\end{array}\right]$ and it is a continuum analog of lattice fractional derivatives that are suggested in these papers. 
Remark 8. It should be noted that an extension of the variational calculus for the Riesz derivatives, which is suggested in [2], is really considered for the so-called Riesz-Riemann-Liouville derivatives and Riesz-Caputo derivatives. In paper [2], the usual Riesz fractional derivatives are not considered. Moreover extensions of variational calculus suggested in [2], are not connected with microstructural lattice approach. The main advantage of our variational approach is that the Riesz-type fractional derivatives are related to the lattice fractional derivatives $[37,39]$.

Remark 9. Using that $(-i)^{2 m}=(-1)^{m}$, the Riesz-type fractional derivatives for even $\alpha=2 m$, where $m \in \mathbb{N}$, are connected with the usual partial derivative of integer orders $2 m$ by the relation

$$
{ }^{R T} \mathbb{D}_{j}^{2 m} f(\mathbf{r})=(-1)^{m} D_{j}^{2 m} f(\mathbf{r})
$$

where we use the notation

$$
D_{j}^{2 m}=\frac{\partial^{2 m}}{\partial x_{j}^{2 m}}
$$

For $\alpha=2$, the Riesz-type derivative is the local operator $-\partial^{2} / \partial x_{j}^{2}$, i.e.,

$$
{ }^{R T} \mathbb{D}_{j}^{2}=-D_{j}^{2}, \quad{ }^{R T} \mathbb{D}_{j}^{4}=D_{j}^{4}
$$

and so on. The fractional derivatives $R T \mathbb{D}_{j}^{2 m}$ for even orders $\alpha$ are local operators. Note that the Riesz derivative ${ }^{R T} \mathbb{D}_{j}^{1}$ cannot be considered as a derivative of the first order with respect to $x_{j}$, i.e., $R T \mathbb{D}_{j}^{1} f(\mathbf{r}) \neq D_{j}^{1} f(\mathbf{r})$. Note that the Riesz-type derivatives of odd orders $\alpha=2 m+1$, where $m \in \mathbb{N}$, are non-local operators that cannot be considered as usual derivatives $D_{j}^{2 m+1}=\partial^{2 m+1} / \partial x_{j}^{2 m+1}$. For $\alpha=1$ the derivative ${ }^{R T} \mathbb{D}_{j}^{1}$ is a nonlocal operator that can be viewed as a "square root of the 1D Laplacian".

\subsection{Integration by parts for Riesz-type fractional derivatives}

Let us now describe a connection of the Riesz-type fractional derivative and the Marchaud fractional derivatives.

Proposition 2. The Riesz-type fractional derivative $R T \mathbb{D}_{x}^{\alpha}$ defined in (7), can be expressed in terms the Marchaud fractional derivatives ${ }^{M} D_{x, \pm}^{\alpha}$ defined in (2), as follows

$$
{ }^{R T} \mathbb{D}_{x}^{\alpha} f(x)=a(\alpha, m)\left(\left({ }^{M} D_{x,+}^{\alpha} f\right)(x)+\left({ }^{M} D_{x,-}^{\alpha} f\right)(x)\right),
$$

where

$$
a(\alpha, m):=\frac{\Gamma(1-\alpha) A_{m}(\alpha)}{\alpha d_{1}(m, \alpha)} .
$$

Proof. The right-sided Marchaud fractional derivative $\left({ }^{M} D_{x,-}^{\alpha} f\right)(x)$ can be written as

$$
\left({ }^{M} D_{x,-}^{\alpha} f\right)(x):=\frac{\alpha}{\Gamma(1-\alpha)} \frac{1}{A_{m}(\alpha)} \int_{-\infty}^{0} \frac{\left(\Delta_{z}^{m} f\right)(x)}{|z|^{1+\alpha}} d z,
$$

Then the sum of the left-sided and right-sided Marchaud fractional derivatives is given by

$$
\begin{aligned}
\left({ }^{M} D_{x,+}^{\alpha} f\right)(x) & +\left({ }^{M} D_{x,-}^{\alpha} f\right)(x) \\
& =\frac{\alpha}{\Gamma(1-\alpha)} \frac{1}{A_{m}(\alpha)} \int_{-\infty}^{+\infty} \frac{\left(\Delta_{z}^{m} f\right)(x)}{|z|^{1+\alpha}} d z .
\end{aligned}
$$

Using (7) and expression (16), we obtain the relation of the Riesz-type and Marchaud fractional derivatives in the form (13).

Representation (13) and Eq. (6) allow us to prove that the integration by parts transforms the Riesz-type fractional derivatives into themselves. We have the following statement.

Proposition 3. The integration by parts for the Riesztype fractional derivatives (7) has the form

$$
\int_{-\infty}^{+\infty}\left({ }^{R T} \mathbb{D}_{x}^{\alpha} f\right)(x) g(x) d x=\int_{-\infty}^{+\infty} f(x)\left({ }^{R T} \mathbb{D}_{x}^{\alpha} g\right)(x) d x .
$$

Proof. Using (13) and then (6), we get

$$
\begin{aligned}
\int_{-\infty}^{+\infty}\left({ }^{R T} \mathbb{D}_{x}^{\alpha} f\right)(x) g(x) d x= & a(\alpha, m) \int_{-\infty}^{+\infty}\left(\left({ }^{M} \mathbb{D}_{x,+}^{\alpha} f\right)(x)\right. \\
& \left.+\left({ }^{M} \mathbb{D}_{x,-}^{\alpha} f\right)(x)\right) g(x) d x= \\
= & a(\alpha, m) \int_{-\infty}^{+\infty} f(x)\left(\left({ }^{M} \mathbb{D}_{x,-}^{\alpha} g\right) \times(x)+\left({ }^{M} \mathbb{D}_{x,+}^{\alpha} g\right)(x)\right) d x= \\
= & \int_{-\infty}^{+\infty} f(x)^{R T} \mathbb{D}_{x}^{\alpha} g(x) d x .
\end{aligned}
$$

As a result, integration by parts (17) does not change the form of the Riesz-type fractional derivative. Note also that this integration by parts does not change the sign in front of integral.

\section{Variational principle for fractional nonlocal continuum}

Let us now consider a variational approach to describe the $N$-dimensional nonlocal continuum. Equations for 
the fractional nonlocal continuum can be derived as the Euler-Lagrange equation for the action functional

$$
S[\varphi]=\int d t \int d^{N} x \mathcal{L}\left(\varphi, D_{t}^{1} \varphi,{ }^{R T} \mathbb{D}_{j}^{\alpha_{1}} \varphi,{ }^{R T} \mathbb{D}_{j}^{\alpha_{2}} \varphi\right),
$$

where $\mathcal{L}\left(\varphi, D_{t}^{1} \varphi,{ }^{R T} \mathbb{D}_{j}^{\alpha_{1}} \varphi,{ }^{R T} \mathbb{D}_{j}^{\alpha_{2}} \varphi\right)$ is the Lagrangian density that define the $N$-dimensional continuum or the field model, $\varphi=\varphi(\mathbf{r}, t)$ is a scalar field. ${ }^{R T} \mathbb{D}_{j}^{\alpha_{1}}$ and ${ }^{R T} \mathbb{D}_{j}^{\alpha_{2}}$ are the Riesz-type fractional derivatives with respect to $x_{j}$ with $j=1,2, \ldots, N$. Note that $x_{j}$ are dimensionless values in the fractional dynamical models [29]. In general, the action functional (18) can be considered for a bounded region $R \subset \mathbb{R}^{N+1}$ by using the Lagrangian density

$$
\mathcal{L}_{B}=\left\{\begin{array}{l}
\mathcal{L},(\mathbf{r}, t) \in R \\
0,(\mathbf{r}, t) \in \mathbb{R}^{N+1} / R
\end{array}\right.
$$

Let us formulate the principle of stationary action for the functional (18).

Proposition 4. Let the Lagrangian density $\mathcal{L}\left(\varphi, D_{t}^{1} \varphi\right.$, $\left.{ }^{R T} \mathbb{D}_{x}^{\alpha_{1}} \varphi,{ }^{R T} \mathbb{D}_{x}^{\alpha_{2}} \varphi\right)$ be a function with continuous first and second (partial) derivatives with respect to all its arguments, and the function $\varphi=\varphi(r, t)$ has the continuous Riesz-type fractional derivatives of orders $\alpha_{1}>0$ and $\alpha_{2}>0$ with respect to $x_{j}$ with $j=1,2, \ldots, N$; in particular, the function $\varphi$ belongs to the Lizorkin space. Then the holonomic variational equation

$$
\delta S[\varphi]=0,
$$

which describes the principle of stationary action for the functional (18), gives the Euler-Lagrange equation

$$
\begin{gathered}
\frac{\partial \mathcal{L}}{\partial \varphi}-D_{t}^{1}\left(\frac{\partial \mathcal{L}}{\partial D_{t}^{1} \varphi}\right)+\sum_{j=1}^{N}{ }^{R T} \mathbb{D}_{j}^{\alpha_{1}}\left(\frac{\partial \mathcal{L}}{\partial^{R T} \mathbb{D}_{j}^{\alpha_{1}} \varphi}\right) \\
+\sum_{j=1}^{N}{ }^{R T} \mathbb{D}_{j}^{\alpha_{2}}\left(\frac{\partial \mathcal{L}}{{ }^{R T} \mathbb{D}_{j}^{\alpha_{2}} \varphi}\right)=0
\end{gathered}
$$

if we assume that the variations $\delta \varphi$ and $\delta x$ are equal to zero on the boundary $\partial R$ of the region $R \subset \mathbb{R}^{N+1}$.

Proof. The first variation of the action functional (18) with respect to $\varphi(\mathbf{r}, t)$ and its derivatives has the form

$$
\begin{aligned}
\delta S[\varphi]= & \int d t \int d^{N} x \delta \mathcal{L}=\int d t \int d^{N} x\left[\frac{\partial \mathcal{L}}{\partial \varphi} \delta \varphi\right. \\
& +\left(\frac{\partial \mathcal{L}}{\partial D_{t}^{1} \varphi}\right) \delta\left(D_{t}^{1} \varphi\right)+ \\
& +\sum_{j=1}^{N}\left(\frac{\partial \mathcal{L}}{\partial^{R T} \mathbb{D}_{j}^{\alpha_{1}} \varphi}\right) \delta\left({ }^{R T} \mathbb{D}_{j}^{\alpha_{1}} \varphi\right) \\
& \left.+\sum_{j=1}^{N}\left(\frac{\partial \mathcal{L}}{\partial R T \mathbb{D}_{j}^{\alpha_{2}} \varphi}\right) \delta\left({ }^{R T} \mathbb{D}_{j}^{\alpha_{2}} \varphi\right)\right] .
\end{aligned}
$$

If the considered continuum and fields do not contain some non-holonomic constraints, then the fractional derivatives and the variation commute:

$$
\begin{aligned}
\delta\left(D_{t}^{1} \varphi\right) & =D_{t}^{1}(\delta \varphi), \delta\left({ }^{R T} \mathbb{D}_{j}^{\alpha_{1}} \varphi\right) \\
& ={ }^{R T} \mathbb{D}_{j}^{\alpha_{1}}(\delta \varphi), \delta\left({ }^{R T} \mathbb{D}_{j}^{\alpha_{2}} \varphi\right)={ }^{R T} \mathbb{D}_{j}^{\alpha_{2}}(\delta \varphi) .
\end{aligned}
$$

Using integration by parts (17), the variation (21) can be represented in the form

$$
\begin{aligned}
\delta S[\varphi]= & \int d t \int d^{N} x\left[\frac{\partial \mathcal{L}}{\partial \varphi} \delta \varphi-D_{t}^{1}\left(\frac{\partial \mathcal{L}}{\partial D_{t}^{1} \varphi}\right)+\right. \\
& +\sum_{j=1}^{N}{ }^{R T} \mathbb{D}_{j}^{\alpha_{1}}\left(\frac{\partial \mathcal{L}}{\partial R T \mathbb{D}_{j}^{\alpha_{1}} \varphi}\right) \\
& \left.+\sum_{j=1}^{N} R T \mathbb{D}_{j}^{\alpha_{2}}\left(\frac{\partial \mathcal{L}}{\partial R T \mathbb{D}_{j}^{\alpha_{2}} \varphi}\right)\right] \delta \varphi(\mathbf{r}, t) .
\end{aligned}
$$

The principle of stationary action is defined by the holonomic variational equation

$$
\delta S[\varphi]=0 .
$$

As a result, the stationary action principle for fractional nonlocal continuum gives eq. (20) that is the fractional Euler-Lagrange equation for the model described by the Lagrangian density $\mathcal{L}=\mathcal{L}\left(\varphi, D_{t}^{1} \varphi,{ }^{R T} \mathbb{D}_{j}^{\alpha_{1}} \varphi,{ }^{R T} \mathbb{D}_{j}^{\alpha_{2}} \varphi\right)$.

In order to derive equations of motion from variational principles, the integration by parts should be used. It is well-known that for wide class of of fractional-order derivatives the integration by parts transforms the leftsided derivatives into the right-sided and vice versa. To avoid this problem, we proposed the variational principle with the Riesz-type fractional derivatives. As we proved, the integration by parts (17) does not change the form of this fractional-order derivative. 
In the next section we consider this fractional variational approach to a model of waves in anisotropic fractional nonlocal media and for a fractional generalization of vibrating string model.

\section{Application of the variational principle to waves in anisotropic fractional nonlocal media}

4.1 Continuum model of waves in fractional nonlocal media

Let us consider waves in anisotropic medium with spatial dispersion of power-law type, i.e., nonlocality of fractional order. We will describe the medium by a scalar field $\varphi=$ $\varphi(\mathbf{r}, t)$ in the Euclidean space $(\mathbf{r}, t) \in \mathbb{R}^{N} \times \mathbb{R}$. The order of non-locality along different directions (along the $x_{j}$-axis) will be denoted by $\alpha_{j} / 2>0$. The Lagrangian density for this field is

$$
\begin{aligned}
\mathcal{L}\left(\varphi, D_{t}^{1} \varphi,{ }^{R T} \mathbb{D}_{j}^{\alpha_{j}} \varphi\right)= & \frac{1}{2} \rho\left(D_{t}^{1} \varphi(\mathbf{r}, t)\right)^{2} \\
& -\frac{1}{2} \sum_{j=1}^{N} A_{j}\left({ }^{R T} \mathbb{D}_{j}^{\alpha_{j} / 2} \varphi(\mathbf{r}, t)\right)^{2} \\
& -V(\varphi(\mathbf{r}, t))
\end{aligned}
$$

If $\alpha_{j}=2$ for all $j=1,2, \ldots, N$, this Lagrangian density defines usual (local) classical field theory.

For the Lagrangian density (23), we get

$$
\begin{aligned}
& \frac{\partial \mathcal{L}}{\partial \varphi}=-\frac{\partial V(\varphi)}{\partial \varphi}, \quad \frac{\partial \mathcal{L}}{\partial\left(D_{t}^{1} \varphi(\mathbf{r}, t)\right)}=\rho D_{t}^{1} \varphi(\mathbf{r}, t), \\
& \frac{\partial \mathcal{L}}{\partial\left(R T \mathbb{D}_{j}^{\alpha_{j} / 2} \varphi(\mathbf{r}, t)\right)}=-A_{j}^{R T} \mathbb{D}_{j}^{\alpha_{j} / 2} \varphi(\mathbf{r}, t) .
\end{aligned}
$$

Substitution of (24) and (25) into (20) gives the EulerLagrange equation of the form

$$
\rho D_{t}^{2} \varphi(\mathbf{r}, t)+\sum_{j=1}^{N} R T \mathbb{D}_{j}^{\alpha_{j} / 2}\left(A_{j}^{R T} \mathbb{D}_{j}^{\alpha_{j} / 2} \varphi(\mathbf{r}, t)\right)+\frac{\partial V(\varphi)}{\partial \varphi}=0,
$$

which is the fractional differential equation for the fractional nonlocal anisotropic medium.

For homogeneous media, $A_{j}$ are independent of $\mathbf{r}$, and the fractional differential Eq. (26) can be rewritten in the form

$$
\rho D_{t}^{2} \varphi(\mathbf{r}, t)+\sum_{j=1}^{N} A_{j}\left({ }^{R T} \mathbb{D}_{j}^{\alpha_{j} / 2}\right)^{2} \varphi(\mathbf{r}, t)=-\frac{\partial V(\varphi)}{\partial \varphi},
$$

For wide class of functions $\varphi(\mathbf{r}, t)$ from the Lizorkin space [11] for example, Eq. (27) can be represented in the form

$$
\rho D_{t}^{2} \varphi(\mathbf{r}, t)+\sum_{j=1}^{N} A_{j}^{R T} \mathbb{D}_{j}^{\alpha_{j}} \varphi(\mathbf{r}, t)=-\frac{\partial V(\varphi)}{\partial \varphi} .
$$

In the case $A_{j}=E$ and $\alpha_{j}=\alpha$ for all $j=1,2, \ldots, N$, Eq. (28) can be written as

$$
\rho D_{t}^{2} \varphi(\mathbf{r}, t)+E^{R T} \Delta^{\alpha} \varphi(\mathbf{r}, t)=-\frac{\partial V(\varphi)}{\partial \varphi},
$$

where

$$
{ }^{R T} \Delta^{\alpha}:=\sum_{j=1}^{N} R T \mathbb{D}_{j}^{\alpha}
$$

is the fractional Laplacian of the Riesz type [37]. Equation (29) describes waves in isotropic nonlocal medium. For $\alpha=2$ and $V(\varphi)=0$, Eq. (29) is the classical wave equation since ${ }^{R T} \Delta^{\alpha}=-\Delta$.

\subsection{Lattice model of waves in fractional nonlocal media}

Let us consider an $N$-dimensional unbounded lattice with the non-coplanar vectors $\mathbf{a}_{j},(j=1,2, \ldots, N)$ that define the distance $a_{j}=\left|\mathbf{a}_{j}\right|$ between particles with mass $M$. For simplification, we will consider mutually orthogonal vectors $\mathbf{a}_{j}$. In the general case, the Cartesian coordinate system does not depend on the choice of lattice vectors $\mathbf{a}_{j}$. However, conveniently choose the basis vectors $\mathbf{e}_{j}$ of the Cartesian coordinate system such that $\mathbf{a}_{j}=a_{j} \mathbf{e}_{j}$. Sites of this lattice will be characterized by the number vector $\mathbf{n}=\left(n_{1}, n_{2}, \ldots, n_{N}\right)$, where $n_{j}(j=1,2, \ldots, N)$ are integers. We assume that the positions of particles in the lattice coincide with the lattice sites, so that the vector $\mathbf{n}$ is a number vector of the corresponding particle.

Let us consider a model of lattice with long-range interactions that is described by the Lagrangian

$$
\begin{aligned}
L\left(\varphi_{\mathbf{n}}(t), \dot{\varphi}_{\mathbf{n}}(t)\right)= & \frac{M}{2} \sum_{\mathbf{n}} \dot{\varphi}_{\mathbf{n}}(t)-\frac{1}{2} \sum_{\mathbf{n}, \mathbf{m}} \sum_{j=1}^{N} \frac{1}{a_{j}^{\alpha}} A_{j} \varphi_{\mathbf{n}}(t) \\
& \times K_{\alpha_{j}}^{+}\left(n_{j}-m_{j}\right) \varphi_{\mathbf{m}}(t)-\sum_{\mathbf{n}} V\left(\varphi_{\mathbf{n}}(t)\right),
\end{aligned}
$$

with the kernel

$$
\begin{aligned}
K_{\alpha_{j}}^{+}\left(n_{j}-m_{j}\right)= & \frac{\pi^{\alpha_{j}}}{\alpha_{j}+1}{ }_{1} F_{2}\left(\frac{\alpha_{j}+1}{2} ; \frac{1}{2}, \frac{\alpha_{j}+3}{2} ;\right. \\
& \left.-\frac{\pi^{2}\left(n_{j}-m_{j}\right)^{2}}{4}\right), \quad \alpha_{j}>0,
\end{aligned}
$$

where ${ }_{1} F_{2}$ is the Gauss hypergeometric function [7]. The first term of (31) defines the kinetic energy. The second and third terms give the potential energy. If we consider $V\left(\varphi_{\mathbf{n}}(t)\right)=J_{\mathbf{n}}(t) \varphi_{\mathbf{n}}(t)$, where $J_{\mathbf{n}}(t)$ is the external force, then we get the linear theory. For $V\left(\varphi_{\mathbf{n}}(t)\right)=(\lambda / 4) \varphi_{\mathbf{n}}^{4}(t)$, we have the well-known nonlinear $\varphi^{4}$-theory. 
The equation of motion can be obtained, as usual, from the stationary condition for action

$$
S_{L}[\varphi]=\int d t L\left(\varphi_{\mathbf{n}}(t), \dot{\varphi}_{\mathbf{n}}(t)\right)
$$

The stationary condition for the functional (33) with the Lagrangian (31) gives the Euler-Lagrange equation

$$
\frac{\partial L}{\partial \varphi_{\mathbf{n}}}-\frac{d}{d t}\left(\frac{\partial \mathcal{L}}{\partial \dot{\varphi}_{\mathbf{n}}}\right)=0
$$

in the form

$M \frac{d^{2} \varphi_{\mathbf{n}}(t)}{d t^{2}}+\sum_{\mathbf{m}} \sum_{j=1}^{N} \frac{1}{a_{j}^{\alpha}} A_{j} K_{\alpha_{j}}^{+}\left(n_{j}-m_{j}\right) \varphi_{\mathbf{m}}(t)=F\left(\varphi_{\mathbf{n}}(t)\right)$,

where $F$ is the potential force

$$
F\left(\varphi_{\mathbf{n}}\right)=-\frac{\partial V\left(\varphi_{\mathbf{n}}\right)}{\partial \varphi_{\mathbf{n}}}
$$

In papers [37, 39], definition of lattice fractional derivatives of the positive real orders $\alpha_{j}$ in the directions $\mathbf{e}_{j}=$ $\mathbf{a}_{j} /\left|\mathbf{a}_{j}\right|$ are presented.

Definition 3. Lattice fractional partial derivatives of orders $\alpha_{j}>0$ are operators defined by the equations

$$
\begin{gathered}
\mathbb{D}_{L}^{+}\left[\begin{array}{c}
\alpha_{j} \\
j
\end{array}\right] \varphi_{\boldsymbol{n}}(t):=\frac{1}{a_{j}^{\alpha}} \sum_{m_{j}=-\infty}^{+\infty} K_{\alpha_{j}}^{+}\left(n_{j}-m_{j}\right) \varphi_{\boldsymbol{m}}(t), \\
(j=1, \ldots, N),
\end{gathered}
$$

where $\alpha_{j} \in \mathbb{R}, \alpha_{j}>0, n_{j}, m_{j} \in \mathbb{Z}, \boldsymbol{m} \in \mathbb{Z}^{3}$, and the interaction kernel $K_{\alpha_{j}}^{+}\left(n_{j}-m_{j}\right)$ is defined by Eq. (32).

Using (37), the Euler-Lagrange Eq. (35) can be rewritten as

$$
M \frac{d^{2} \varphi_{\mathbf{n}}(t)}{d t^{2}}+\sum_{\mathbf{m}} \sum_{j=1}^{N} A_{j} \mathbb{D}_{L}^{+}\left[\begin{array}{c}
\alpha_{j} \\
j
\end{array}\right] \varphi_{\mathbf{m}}(t)=F\left(\varphi_{\mathbf{n}}(t)\right)
$$

There is a connection between the lattice fractional derivatives and the Riesz-type fractional derivatives that is described by the following proposition established in [37, 39].

Proposition 5. In the continuum limit the lattice fractional derivatives (37) are transformed into the continuum fractional derivatives of orders $\alpha_{j}$ that are the Riesz-type fractional derivatives with respect to coordinates $x_{j}$ by

$$
\mathcal{F}^{-1} \circ \operatorname{Lim} \circ \mathcal{F}_{\Delta}\left(\mathbb{D}_{L}^{+}\left[\begin{array}{c}
\alpha_{j} \\
j
\end{array}\right]\right)={ }^{R T} \mathbb{D}_{j}^{\alpha_{j}}
$$

where $\mathcal{F}_{\Delta}$ is the Fourier series transform, Lim is the passage to the limit $a_{j} \rightarrow 0$, and $\mathcal{F}^{-1}$ is the inverse Fourier integral transform [39].

Proof. This proposition is proved in Section 5 of [39].

Using this proposition, we can get that the continuum limit transforms of lattice Eqs. (38) into (28), which is the fractional partial differential equation of the fractional nonlocal continuum.

As a result, proposed fractional variational principle allows us to get the Euler-Lagrange equations that are directly connected with microstructural lattice models of fractional nonlocal media [31-34, 40], and the lattice field theories [35, 38].

\section{Application of the variational principle to fractional nonlocal one-dimensional continuum}

5.1 Continuum and lattice models of string with fractional nonlocality

Let us now consider a string, which is made of a material with spatial dispersion of power-law type. The Lagrangian density of this string with nonlocality of two orders $\alpha_{1}>0$ and $\alpha_{2}>0$ can be presented in the form

$$
\begin{aligned}
\mathcal{L}\left(\varphi, D_{t}^{1} \varphi,{ }^{R T} \mathbb{D}_{x}^{\alpha_{1}} \varphi,{ }^{R T} \mathbb{D}_{x}^{\alpha_{2}} \varphi\right)= & \frac{1}{2} \rho\left(D_{t}^{1} \varphi(x, t)\right)^{2} \\
& -\frac{1}{2} T\left({ }^{R T} \mathbb{D}_{x}^{\alpha_{1}} \varphi(x, t)\right)^{2} \\
& -\frac{1}{2} T l_{s}^{2}\left(\alpha_{2}\right)\left({ }^{R T} \mathbb{D}_{x}^{\alpha_{2}} \varphi(x, t)\right)^{2} \\
& +J(x, t) \varphi(x, t) .
\end{aligned}
$$

For an incompressible elastic solid, the displacement field $\varphi(x)=u_{y}(x)$ is transversal, or orthogonal to the longitudinal axis (in our case, the $x$-axis) of wave propagation. Note that $x$ and $l_{s}^{2}\left(\alpha_{2}\right)$ for fractional nonlocal models are dimensionless values [29]. The first term represents the kinetic energy, where $\rho$ is the linear density (i.e., mass per unit length). The second and third terms represent the potential energy due to internal forces, and $T$ is the string tension. The fourth term represents the potential energy due to the external load $J(x)$. 
For local materials, we have $\alpha_{1}=1, \alpha_{2}=2$, and we should use the Lagrangian density in the form

$$
\begin{aligned}
\mathcal{L}\left(\varphi, D_{t}^{1} \varphi, D_{x}^{2} \varphi, D_{x}^{3} \varphi\right)= & \frac{1}{2} \rho\left(D_{t}^{1} \varphi(x, t)\right)^{2}-\frac{1}{2} T\left(D_{x}^{1} \varphi(x, t)\right)^{2}+ \\
& +\frac{1}{2} T l_{s}^{2}\left(D_{x}^{2} \varphi(x, t)\right)^{2}+J(x, t) \varphi(x, t) .
\end{aligned}
$$

Note that the Lagrangian density (40) with $\alpha_{1}=1$ and $\alpha_{2}=2$ similar to (41) but contains minus sign in front of the third term. Therefore expression (40) cannot give (41) for $\alpha_{1}=1, \alpha_{2}=2$.

For the Lagrangian density (40) of the string model, we get the derivatives

$$
\begin{gathered}
\frac{\partial \mathcal{L}}{\partial \varphi}=J(x, t), \quad \frac{\partial \mathcal{L}}{\partial D_{t}^{1} \varphi(x, t)}=\rho D_{t}^{1} \varphi(x, t), \\
\frac{\partial \mathcal{L}}{\partial^{R T} \mathbb{D}_{x}^{\alpha_{1}} \varphi(x, t)}=-T^{R T} \mathbb{D}_{x}^{\alpha_{1}} \varphi(x, t), \\
\frac{\partial \mathcal{L}}{\partial^{R T} \mathbb{D}_{x}^{\alpha_{2}} \varphi(x, t)}=-T l_{s}^{2}\left(\alpha_{2}\right)^{R T} \mathbb{D}_{x}^{\alpha_{2}} \varphi(x, t) .
\end{gathered}
$$

Substitution of (42) and (43) into (20) gives the EulerLagrange equation

$$
\begin{aligned}
\rho D_{t}^{2} \varphi & +{ }^{R T} \mathbb{D}_{x}^{\alpha_{1}}\left(T\left({ }^{R T} \mathbb{D}_{x}^{\alpha_{1}}\right) \varphi\right) \\
& +{ }^{R T} \mathbb{D}_{x}^{\alpha_{2}}\left(T l_{s}^{2}\left(\alpha_{2}\right)^{R T} \mathbb{D}_{x}^{\alpha_{2}} \varphi\right)-J(x, t)=0
\end{aligned}
$$

which is the fractional differential equation for the fractional nonlocal string.

If the continuum is homogeneous, then $T$ is independent of $x$, and the fractional string equation has simple form

$\rho D_{t}^{2} \varphi+T\left({ }^{R T} \mathbb{D}_{x}^{\alpha_{1}}\right)^{2} \varphi+T l_{s}^{2}\left(\alpha_{2}\right)\left({ }^{R T} \mathbb{D}_{x}^{\alpha_{2}}\right)^{2} \varphi-J(x, t)=0$.

Using the properties of the fractional Riesz derivatives, for functions $\varphi(x)$, for which $\left({ }^{R T} \mathbb{D}_{x}^{\alpha_{2}}\right)^{2} \varphi={ }^{R T} \mathbb{D}_{x}^{2 \alpha_{1}} \varphi$, Eq. (45) can be represented in the form

$$
\rho D_{t}^{2} \varphi+T^{R T} \mathbb{D}_{x}^{2 \alpha_{1}} \varphi+T l_{s}^{2}\left(\alpha_{2}\right)^{R T} \mathbb{D}_{x}^{2 \alpha_{2}} \varphi-J(x, t)=0 .
$$

In the general case, we should consider an effective source term $J_{\text {eff }}(x, t)$ instead of $J(x, t)$, where $J_{\text {eff }}(x, t)$ contains the function $J(x, t)$ and deviations from the semigroup property for the Riesz-type derivatives.
It should be noted that fractional differential Eq. (46) is directly connected with the lattice model described by the equation

$$
\begin{aligned}
\rho \frac{d^{2} \varphi_{n}(t)}{d t^{2}} & +\frac{T}{a^{2 \alpha_{1}}} \sum_{m} K_{2 \alpha_{1}}^{+}(n-m) \varphi_{m}(t) \\
& +\frac{T l_{s}^{2}\left(\alpha_{2}\right)}{a^{2 \alpha_{2}}} \sum_{m} K_{2 \alpha_{2}}^{+}(n-m) \varphi_{m}(t)-J_{n}(t)=0,
\end{aligned}
$$

that can be derived from stationary action principle with the Lagrangian

$$
\begin{aligned}
L\left(\varphi_{n}(t), \dot{\varphi}_{n}(t)\right)= & \frac{\rho}{2} \sum_{n} \dot{\varphi}_{n}(t) \\
& -\frac{T}{2 a^{2 \alpha_{1}}} \sum_{n, m} \varphi_{n}(t) K_{2 \alpha_{1}}^{+}(n-m) \varphi_{m}(t)- \\
& -\frac{T l_{s}^{2}\left(\alpha_{2}\right)}{2 a^{2 \alpha_{2}}} \sum_{n, m} \varphi_{n}(t) K_{2 \alpha_{2}}^{+}(n-m) \varphi_{m}(t) \\
& -\sum_{n} J_{n}(t) \varphi_{n}(t),
\end{aligned}
$$

where the kernel $K_{\alpha}^{+}(n-m)$ is defined by Eq. (32).

If we have materials without nonlocality and memory, then $\alpha_{1}=1, \alpha_{2}=2$, and Eq. (46) has the form

$$
\rho D_{t}^{2} \varphi-T D_{x}^{2} \varphi+T l_{s}^{2} D_{x}^{4} \varphi-J(x, t)=0,
$$

where we use ${ }^{R T} \mathbb{D}_{x}^{2}=-D_{x}^{2}$, and ${ }^{R T} \mathbb{D}_{x}^{4}=+D_{x}^{4}$.

Note that despite the fact that the Lagrangian density (40) cannot give Lagrangian density (41) for $\alpha_{1}=1, \alpha_{2}=$ 2 , the eqs. (46) gives (49) for $\alpha_{1}=1, \alpha_{2}=2$. This is due to the fact that

$$
\begin{aligned}
& \frac{\partial}{\partial^{R T} \mathbb{D}_{x}^{2} \varphi(x, t)}=-\frac{\partial}{\partial D_{x}^{2} \varphi(x, t)}, \\
& \frac{\partial}{\partial^{R T} \mathbb{D}_{x}^{4} \varphi(x, t)}=+\frac{\partial}{\partial D_{x}^{4} \varphi(x, t)} .
\end{aligned}
$$

Equation (49) is the differential equation for string which was made of a gradient elasticity material $[16,17$, 36,41 ]. If we use $\alpha_{1}=1, \alpha_{2}=0$, we get the equation

$$
\rho D_{t}^{2} \varphi-T D_{x}^{2} \varphi-J(x, t)=0 .
$$

It is the usual well-known equation for string without nonlocality and memory.

\subsection{Solution of fractional differential equation}

For the static case $\left(D_{t}^{2} \varphi=0\right.$ and $\left.J(x, t)=J(x)\right)$, Eq. (46) has the form

$$
{ }^{R T} \mathbb{D}_{x}^{2 \alpha_{1}} \varphi+l_{s}^{2}\left(\alpha_{2}\right)^{R T} \mathbb{D}_{x}^{2 \alpha_{2}} \varphi=T^{-1} J(x) .
$$


The particular solution of Eq. (52) can be presented (see Corollary 5.14 of [11]) in the form

$$
\varphi(x)=T^{-1} \int_{-\infty}^{+\infty} G_{2 \alpha_{1}, 2 \alpha_{2}}\left(x-x^{\prime}\right) J\left(x^{\prime}\right) d x^{\prime},
$$

where $G_{\alpha_{1}, \alpha_{2}}(x)$ is the Green-type function

$$
G_{2 \alpha_{1}, 2 \alpha_{2}}(x)=\int_{0}^{\infty} \frac{\cos (\lambda|x|)}{\lambda^{2 \alpha_{1}}+l_{s}^{2}\left(\alpha_{2}\right) \lambda^{2 \alpha_{2}}} d \lambda .
$$

If the external force $J(x)$ is applied at the point $x=0$, then

$$
J(x)=J_{0} \delta(x)
$$

and the field $\varphi(x)$ has a simple form $\varphi(x)=$ $\left(J_{0} / T\right) G_{2 \alpha_{1}, 2 \alpha_{2}}(x)$, where $G_{2 \alpha_{1}, 2 \alpha_{2}}(x)$ is defined by $(54)$. As a result, the field $\varphi(x)$ is given by

$$
\varphi(x)=\frac{2 J_{0}}{\pi T} \int_{0}^{\infty} \frac{\cos (\lambda|x|)}{\lambda^{2 \alpha_{1}}+l_{s}^{2}\left(\alpha_{2}\right) \lambda^{2 \alpha_{2}}} d \lambda
$$

The plane wave

$$
\varphi(x, t)=e^{-i \omega t} \varphi_{p}(x),
$$

can be traveling in fractional nonlocal continua with its frequency $\omega$, where the fractional differential equation for the field $\varphi_{p}(x)$ is

$$
\begin{aligned}
-\rho \omega^{2} \varphi_{p}(x) & +T\left({ }^{R T} \mathbb{D}_{x}^{\alpha_{1}}\right)^{2} \varphi_{p}(x) \\
& +T l_{s}^{2}\left(\alpha_{2}\right)\left({ }^{R T} \mathbb{D}_{x}^{\alpha_{2}}\right)^{2} \varphi_{p}(x)-J_{p}(x)=0 .
\end{aligned}
$$

Here we use $J(x, t)=e^{-i \omega t} J_{p}(x)$. For a wide class of functions $\varphi_{p}(x) \mathrm{Eq}$. (57) can be rewritten in the form

$$
\begin{aligned}
R T \mathbb{D}_{x}^{2 \alpha_{1}} \varphi_{p}(x) & +l_{s}^{2}\left(\alpha_{2}\right)^{R T} \mathbb{D}_{x}^{2 \alpha_{2}} \varphi_{p}(x) \\
& -\frac{\rho \omega^{2}}{T} \varphi_{p}(x)=T^{-1} J_{p}(x) .
\end{aligned}
$$

The particular solution of Eq. (58) can be given (Theorem 5.24 of [11]) in the form

$$
\varphi_{p}(x, \omega)=T^{-1} \int_{-\infty}^{+\infty} G_{2 \alpha_{1}, 2 \alpha_{2}}\left(x-x^{\prime}, \omega\right) J_{p}\left(x^{\prime}\right) d x^{\prime},
$$

where $G_{2 \alpha_{1}, 2 \alpha_{2}}^{(\omega)}(x)$ is the Green-type function

$$
G_{2 \alpha_{1}, 2 \alpha_{2}}(x, \omega)=2 \int_{0}^{\infty} \frac{\cos (\lambda|x|)}{\lambda^{2 \alpha_{1}}+l_{s}^{2}\left(\alpha_{2}\right) \lambda^{2 \alpha_{2}}-\rho \omega^{2} / T} d \lambda .
$$

Here $l_{s}^{2}\left(\alpha_{2}\right) \neq 0$ and $\rho \omega^{2} \neq 0$. For the case (55), the solution (59) is

$$
\varphi_{p}(x, \omega)=\frac{2 J_{0}}{T} \int_{0}^{\infty} \frac{\cos (\lambda|x|)}{\lambda^{2 \alpha_{1}}+l_{s}^{2}\left(\alpha_{2}\right) \lambda^{2 \alpha_{2}}-\rho \omega^{2} / T} d \lambda .
$$

This is solution of the fractional string equation for external load $J(x)$ applied at a point (55).

\section{Conclusion}

To obtain equations of fractional nonlocal theory for media and fields with power-law nonlocality, we propose a new fractional variational principle for Lagrangians with fractional-order derivatives. This fractional variational principle is based on the Riesz-type fractional derivatives. The characteristic properties of the proposed approach are the following:

(1) The Riesz-type of derivatives do not have the left-sided and the right-sided forms, in contrast to the Riemann-Liouville, Caputo, Liouville and Marchaud fractional derivatives.

(2) The integration by parts transforms the Riesz-type fractional derivatives into themselve. For fractional derivatives of Riemann-Liouville, Caputo, Liouville, and Marchaud, the integration by parts, which should be used in derivation of the Euler-Lagrange equations from variational principle, transforms the left-sided derivatives into the right-sided and vice versa.

(3) The corresponding fractional Euler-Lagrange equations can be solved for a wide class of Lagrangian densities by methods described in [11].

(4) The Riesz-type fractional derivative ${ }^{R T} \mathbb{D}_{j}^{\alpha_{j}}$ is a derivative with respect to one coordinate $x_{j} \in \mathbb{R}^{1}$ contrary to the usual Riesz derivative [11, 23], which is a fractional generalization of $N$-dimensional Laplacian. The Riesz-type derivative ${ }^{R T} \mathbb{D}_{j}^{\alpha_{j}}$ can be viewed as a partial derivative of non-integer order.

(5) The Riesz-type fractional derivatives naturally arise in the fractional continuum mechanics based on the lattice models [31-34, 40], since they directly connected with lattice fractional derivatives that are recently proposed $[37,39]$.

The main advantage of the suggested fractional variational principle is the fact that it is connected with microstructural lattice approach and the lattice fractional calculus that is recently proposed. In the papers [37, 39] it was proves that the Riesz-type fractional derivative is a continuum limit of the lattice fractional derivative.

Nonlocal continuum theory is based on the assumption that the forces between particles of continuum have long-range type, thus reflecting the long-range character of inter-atomic forces. Nonlocality of the power-law type allows us to use fractional derivatives and integrals in nonlocal continuum mechanics. In fractional nonlocal theory it is important to apply fractional derivatives that take into account the long-range particle interactions in microstructural models. The microstructural lattice approach, which includes consideration of continuum 
limit, allows us to select a type of fractional derivatives that will be more adequately and correctly describe fractional nonlocal continua. Therefore we propose to use the Riesz type fractional-order derivatives that are directly connected with models of lattices with long-range interactions. It allow us to get more correct models of fractional nonlocal media by fractional variational principle.

In this paper we consider a fractional principle of stationary action with Riesz-type fractional derivatives. This principle is represented by the holonomic variational equation $\delta S[\varphi]=0$. In the general case, we should use the variational principles that are represented by nonholonomic variational equations. To consider nonlocal media with dissipation and non-potential forces, nonLagrangian systems, we should apply a fractional generalization of the Sedov non-holonomic variational equation [24-26] instead of the stationary action principle.

The proposed fractional variational principle also allows us to obtain exact analytical solutions of the fractional differential equations for models of a wide class of media with fractional nonlocality. A characteristic feature of the behavior of a fractional nonlocal continuum is the spatial power-tails of non-integer orders [12,33]. The fractional nonlocal models, which are used to describe complex media, can be characterized by a common or universal spatial behavior media by analogy with the universal temporal behavior of low-loss dielectrics [9, 10, 27, 28]. The proposed fractional variational principle can be important in the fractional field theory [38] and in the fractional quantum theory [35].

\section{Acknowledgments}

I would like to express my sincere appreciation to Reviewers for valuable comments and Editors and Editorial Staff for successful collaboration and excellent work.

Received: 29 March 2015 Revised: 24 August 2015

Accepted: 27 August 2015

Published online: 04 September 2015

\section{References}

1. Agrawal, O.P.: Formulation of Euler-Lagrange equations for fractional variational problems, pp. 368-379, (2002)

2. Agrawal, O.P.: Fractional variational calculus in terms of Riesz fractional derivatives. J. Phys. A. 24, 6287-6303 (2007)

3. Agrawal, O.P.: Generalized multiparameters fractional variational calculus. Int. J. Differential Equations. 2012, 521750 (2012)

4. Almeida, R., Torres, D.F.M.: Calculus of variations with fractional derivatives and fractional integrals. Appl. Math. Lett. 22(12), 1816-1820 (2009). (arXiv:0907.1024)

5. Almeida, R., Malinowska, A.B., Torres, D.F.M.: A fractional calculus of variations for multiple integrals with application to vibrating string. J. Math. Phys. 51(3), 033503 (2010). (arXiv:1001.2722)

6. Atanackovic, T.M., Pilipovic, S., Stankovic, B., Zorica, D.: Fractional Calculus with Applications in Mechanics: Wave Propagation, Impact and Variational Principles. Wiley-ISTE, London, Hoboken (2014)

7. Erdelyi, A., Magnus, W., Oberhettinger, F., Tricomi, F.G.: Higher Transcendental Functions Volume 1. McGraw-Hill, New York, (1953), and Krieeger, Melbourne, Florida, (1981)

8. Eringen, A.C.: Nonlocal Continuum Field Theories. Springer, New York (2002)
9. Jonscher, A.K.: The universal dielectric response. Nature. 267, 673-679 (1977)

10. Jonscher, A.K.: Universal Relaxation Law. Chelsea Dielectrics, London (1996)

11. Kilbas, A.A., Srivastava, H.M., Trujillo, J.J.: Theory and Applications of Fractional Differential Equations (2006)

12. Korabel, N., Zaslavsky, G.M., Tarasov, V.E.: Coupled oscillators with powerlaw interaction and their fractional dynamics analogues. Commun. Nonlin. Sci. Numeric. Simul. 12(8), 1405-1417 (2007). (arXiv:math-ph/0603074)

13. Mainardi, F.: Fractional calculus: Some basic problems in continuum and statistical mechanics. In: Carpinteri, A., Mainardi F (eds.) Fractals and Fractional Calculus in Continuum Mechanics, pp. 291-348. Springer, Wien and New York, (1997). (arXiv:1201.0863)

14. Mainardi, F.: Fractional Calculus and Waves in Linear Viscoelasticity: An Introduction to Mathematical Models. World Scientific, Singapore (2010)

15. Malinowska, A.B., Torres, D.F.M.: Fractional calculus of variations for a combined Caputo derivative. Fractional Calculus Appl. Anal. 14(4), 523-537 (2011)

16. Mindlin, R.D.: Micro-structure in linear elasticity. Arch. Rational Mech. Anal. 16(1), 51-78 (1964)

17. Mindlin, R.D.: Theories of elastic continua and crystal lattice theories. In: Kroner, E. (ed.) Mechanics of Generalized Continua, pp. 312-320. Springer-Verlag, Berlin, (1968)

18. Nasrolahpour, H.: Fractional Lagrangian and Hamiltonian formulations in field theory Generalized multiparameters fractional variational calculus. Prespacetime J. 4(3), 604-608 (2013)

19. Odzijewicz, T., Malinowska, AB., Torres, D. F. M.: Fractional variational valculus with vlassical and vombined Caputo derivatives. Nonlinear Anal. 75(3), 1507-1515 (2012). (arXiv:1101.2932)

20. Riesz, M.: L'intégrale de Riemann-Liouville et le probléme de Cauchy. Acta Math. 81(1), 1-222 (1949). in French

21. Rogula, D.: Nonlocal Theory of Material Media. Springer-Verlag, New York (1983)

22. Sabatier, J., Agrawal, O.P., Tenreiro Machado, J.A. (Eds): Advances in Fractional Calculus. Theoretical Developments and Applications in Physics and Engineering. Springer, Dordrecht (2007)

23. Samko, S.G., Kilbas, A.A., Marichev, O.I.: Integrals and Derivatives of Fractional Order and Applications (Nauka i Tehnika, Minsk, 1987); and Fractional Integrals and Derivatives Theory and Applications Gordon and Breach, New York (1993)

24. Sedov, L.I.: Mathematical methods for constructing new models of continuous media. Russ. Math. Surv. 20(5), 123-182 (1965)

25. Sedov, L.I.: Models of continuous media with internal degrees of freedom. J. Appl. Math. Mech. 32(5), 803-819 (1968)

26. Sedov, L.I., Tsypkin, A.G.: Principles of the Microscopic Theory of Gravitation and Electromagnetism, Nauka, Moscow (1989). in Russian

27. Tarasov, V.E.: Universal electromagnetic waves in dielectrics. J. Phys: Condensed Matter. 20(17), 175223 (2008). (arXiv:0907.2163)

28. Tarasov, V.E.: Fractional integro-differential equations for electromagnetic waves in dielectric media. Theor. Math. Phys. 158(3), 355-359 (2009). (arXiv:1 107.5892)

29. Tarasov, V.E.: Fractional Dynamics: Applications of Fractional Calculus to Dynamics of Particles, Fields and Media. Springer, New York (2011)

30. Tarasov, V.E.: Review of some promising fractional physical models. Int. J. Modern Phys. B. 27(9), 1330005 (2013). (arXiv:1502.07681)

31. Tarasov, V.E.: Lattice model with power-law spatial dispersion for fractional elasticity. Central Eur. J. Phys. 11(11), 1580-1588 (2013). (arXiv:1501.01201)

32. Tarasov, V.E.: Lattice model of fractional gradient and integral elasticity: Long-range interaction of Grünwald-Letnikov-Riesz type. Mech. Mater. 70(1), 106-114 (2014). (arXiv:1502.06268)

33. Tarasov, V.E.: Lattice with long-range interaction of power-law type for fractional non-local elasticity. Int. J. Solids Struct. 51, 2900-2907 (2014). (arXiv:1502.05492)

34. Tarasov, V.E.: Fractional gradient elasticity from spatial dispersion law. ISRN Condensed Matter Phys. 2014. Article ID 794097, 13 pages (2014). (arXiv:1306.2572)

35. Tarasov, V.E.: Fractional quantum field theory: From lattice to continuum. Adv. High Energy Phys. 2014, 957863 (2014). 14 pages

36. Tarasov, V.E.: General lattice model of gradient elasticity. Modern Phys. Lett. B. 28(7), 1450054 (2014). (arXiv:1501.01435)

37. Tarasov, V.E.: Toward lattice fractional vector calculus. J. Phys. A. 47(35), 355204 (2014). (51 pages) 
38. Tarasov, V.E.: Non-linear fractional field equations: weak non-linearity at power-law non-locality. Nonlinear Dynam. 80(4), 1665-1672 (2015)

39. Tarasov, V.E.: Lattice fractional calculus. Appl. Math. Comput. 257, 12-33 (2015)

40. Tarasov, V.E.: Three-dimensional lattice models with long-range interactions of Grünwald-Letnikov type for fractional generalization of gradient elasticity. Meccanica. 50 (2015). doi:10.1007/s11012-015-0190-4

41. Tarasov, V.E.: Lattice model with nearest-neighbor and

next-nearest-neighbor interactions for gradient elasticity. Discontinuity, Nonlinearity, Complexity. 4(1), 11-23 (2015). (arXiv:1503.03633)

42. Valerio, D., Trujillo, J.J., Rivero, M., Tenreiro Machado, J.A., Baleanu, D. Fractional calculus: A survey of useful formulas. Eur. Phys. J. Spec. Topics. 222(8), 1827-1846 (2013)

43. Zhou, Y.: Basic Theory of Fractional Differential Equations. World Scientific, Singapore (2014)

\section{Submit your manuscript to a SpringerOpen ${ }^{\circ}$ journal and benefit from:}

- Convenient online submission

$\checkmark$ Rigorous peer review

- Immediate publication on acceptance

- Open access: articles freely available online

- High visibility within the field

Retaining the copyright to your article

Submit your next manuscript at $\gg$ springeropen.com 